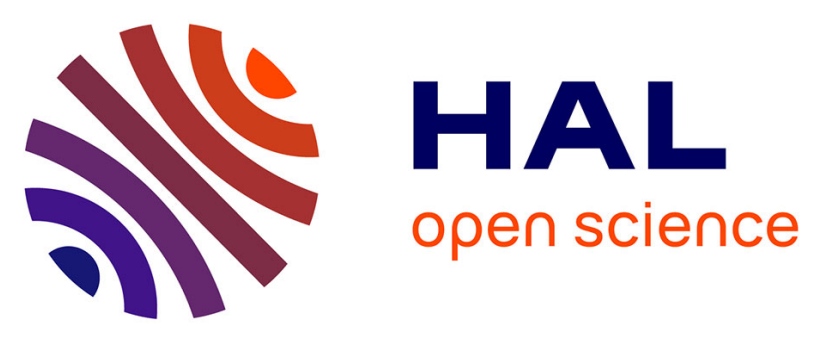

\title{
A cost-effective monitoring technique in particle therapy via uncollimated prompt gamma peak integration
}

J. Krimmer, G. Angellier, L. Balleyguier, D. Dauvergne, N. Freud, J. Herault, Jean Michel Létang, H. Mathez, M. Pinto, E. Testa, et al.

\section{- To cite this version:}

J. Krimmer, G. Angellier, L. Balleyguier, D. Dauvergne, N. Freud, et al.. A cost-effective monitoring technique in particle therapy via uncollimated prompt gamma peak integration. Applied Physics Letters, 2017, 110 (15), pp.154102. 10.1063/1.4980103 . hal-01508408

\section{HAL Id: hal-01508408 \\ https://hal.science/hal-01508408}

Submitted on 14 Apr 2017

HAL is a multi-disciplinary open access archive for the deposit and dissemination of scientific research documents, whether they are published or not. The documents may come from teaching and research institutions in France or abroad, or from public or private research centers.
L'archive ouverte pluridisciplinaire HAL, est destinée au dépôt et à la diffusion de documents scientifiques de niveau recherche, publiés ou non, émanant des établissements d'enseignement et de recherche français ou étrangers, des laboratoires publics ou privés. 


\section{A Cost-Effective Monitoring Technique in Particle Therapy via Uncollimated Prompt}

\section{Gamma Peak Integration}

J. Krimmer, ${ }^{1, a)}$ G. Angellier, ${ }^{2}$ L. Balleyguier, ${ }^{1}$ D. Dauvergne, ${ }^{3,1}$ N. Freud, ${ }^{4}$ J. Hérault, ${ }^{2}$ J.M. Létang, ${ }^{4}$ H. Mathez, ${ }^{1}$ M. Pinto, ${ }^{1, b)}$ E. Testa, ${ }^{1}$ and Y. Zoccarato ${ }^{1}$

1) Institut de Physique Nucléaire de Lyon; Université de Lyon, Université de Lyon 1, IN2P3/CNRS, UMR 5822, F-69622 Villeurbanne cedex, France.

2) Centre Antoine Lacassagne, Cyclotron Biomédical, 227 Avenue de la Lanterne, 06200 Nice, France.

3) Laboratoire de Physique Subatomique et de Cosmologie, CNRS/IN2P3, Université Grenoble-Alpes, UMR 5821 38026 Grenoble cedex, France.

4) Université de Lyon, CREATIS, CNRS UMR 5220, Inserm U1044, INSA - Lyon, Université Lyon 1, Centre Léon Bérard, France.

(Dated: 13 April 2017) 
For the purpose of detecting deviations from the prescribed treatment during particle therapy, the integrals of uncollimated prompt gamma-ray timing distributions are investigated. The intention is to provide information, with a simple and cost-effective setup, independent from monitoring devices of the beamline.

Measurements have been performed with $65 \mathrm{MeV}$ protons at a clinical cyclotron. Prompt gamma-rays emitted from the target are identified by means of time-of-flight. The proton range inside the PMMA target has been varied via a modulator wheel. The measured variation of the prompt gamma peak integrals (PGPI) as a function of the modulator position is consistent with simulations. With detectors covering a solid angle of $25 \mathrm{msr}$ (corresponding to a diameter of 3-4 inches at a distance of $50 \mathrm{~cm}$ from the beam axis) and $10^{8}$ incident protons, deviations of a few per cent in the prompt gamma-ray count rate can be detected. For the present configuration, this change in the count rate corresponds to a $3 \mathrm{~mm}$ change of the proton range in a PMMA target. Furthermore, simulation studies show that a combination of the signals from multiple detectors may be used to detect a misplacement of the target. A different combination of these signals results in a precise number of the detected prompt gamma rays, which is independent on the actual target position.

a) Corresponding author. Email address: jkrimmer@ipnl.in2p3.fr

b) Now with: Ludwig-Maximilians-Universität München, Garching b. München, Germany 


\section{INTRODUCTION}

Tumor treatment with a beam of protons or carbon ions, i.e. particle therapy (PT), is an expanding technology with new clinics being built worldwide. This treatment modality benefits from the fact that a large fraction of the energy from the incident ions is deposited close to the end of the particle path, in the so-called Bragg-peak region. This allows, in comparison to classical radiotherapy with photons, a better conformation of the applied dose to the tumor region with a simultaneous sparing of surrounding healthy tissue. An issue for the quality control of PT is the matching of the Bragg peak location to the target volume. Mispositioning would lead to an under-dosage of the tumor region and an over-dosage in healthy tissue.

Different monitoring approaches exist. The first method which demonstrated clinical applicability $^{1}$ was based on positron emission tomography (PET). With acquisition times in the order of several minutes, however, biological washout needs to be taken into account for a deduction of the absorbed dose via the measured activity distribution.

For the purpose of online monitoring, it has been shown that the generation of prompt gamma-rays is highly correlated to the range of incident ions ${ }^{2}$. The methods under development for an online monitoring comprise mechanically ${ }^{3-6}$ or electronically ${ }^{7-9}$ collimated systems, where the latter are of the Compton camera type. Prototypes based on collimated prompt gamma-ray detection are on the way ${ }^{10,11}$ and have been partially tested clinically at proton therapy centers. In an uncollimated approach ${ }^{12,13}$ the correlation of the mean value and width of time-of-flight (TOF) distributions with the energy and range of incident protons is utilized (prompt-gamma timing technique). This promising method requires, however, an excellent time resolution ( $<1 \mathrm{~ns}$ ) which might already be degraded by the time structure of the beam ${ }^{14}$, especially with the upcoming accelerators of the synchrocyclotron type.

Collimated systems ${ }^{2-6,10,11}$ have already mechanical constraints due to the weight of the collimator, furthermore either a multitude of single detectors, or position sensitive detectors are required. Compton cameras ${ }^{7-9}$ are even more complex in terms of the number of readout channels and an (iterative) image reconstruction is required.

The goal of the present study is to provide a simple and cost-effective monitoring system, which is independent from other monitoring devices of the beamline, as it detects the prompt 
gamma-rays generated inside the patient. The general purpose is to detect deviations of the prescribed treatment, and in particular avoid severe overdosage like in a case, where 63 Gy instead of 3 Gy were applied in a single fraction ${ }^{15}$.

In a previous study ${ }^{16}$ measurements revealed a connection of the ambient dose in the treatment room with the monitoring units (MU) of the beam delivery system. In the present investigation TOF information is used for a discrimination of prompt gamma-rays generated in the target, from those produced in the beamline, e.g. in the nozzle. The quantity to be exploited is the integral in the prompt gamma-ray TOF distributions.

In the following, measurements will be presented, which demonstrate the variation of the PGPI with the variation of the proton range inside a PMMA target via the use of a modulator wheel. Thereafter, it will be discussed, to what extent under clinical conditions and with realistic size detectors, changes in the expected count rate can be detected. Finally, it will be shown, that the combination of signals from multiple detectors placed around a target might be used either to detect a misplacement of the target, or to provide a precise number of the registered prompt gamma-rays, which is independent on the actual target position.

\section{EXPERIMENTAL SETUP}

Experiments have been performed at the Centre Antoine Lacassagne (CAL) in Nice, France. The isochronous cyclotron provides $65 \mathrm{MeV}$ protons for the treatment of eye tumors $^{17}$. In order to cover the whole tumor volume along the beam direction, i.e. to generate a spread out Bragg peak (SOBP), the beam is passing through a modulator wheel, which degrades the fixed energy of the cyclotron. A sketch of the experimental configuration including the main elements of the beamline is given in Figure 1. The modulator wheel used for this experiment is indicated in the insert. It is made from PMMA, the thickness varies from 0 to $14 \mathrm{~mm}$ in steps of $0.8 \mathrm{~mm}$. Ionizing chambers (IC) record the flux of traversing

protons. The axis of the cylindrical PMMA target has been oriented vertically, parallel to the y-axis of the indicated coordinate system. The number of protons hitting the target after traversing the collimator depends on scattering in the modulator wheel and therefore also on the position of the wheel. This transmission has been obtained via simulations and will be detailed in the following section. 
The scintillation detectors present at the experiment comprise cerium doped $\mathrm{LaBr}_{3}(25.4 \times$

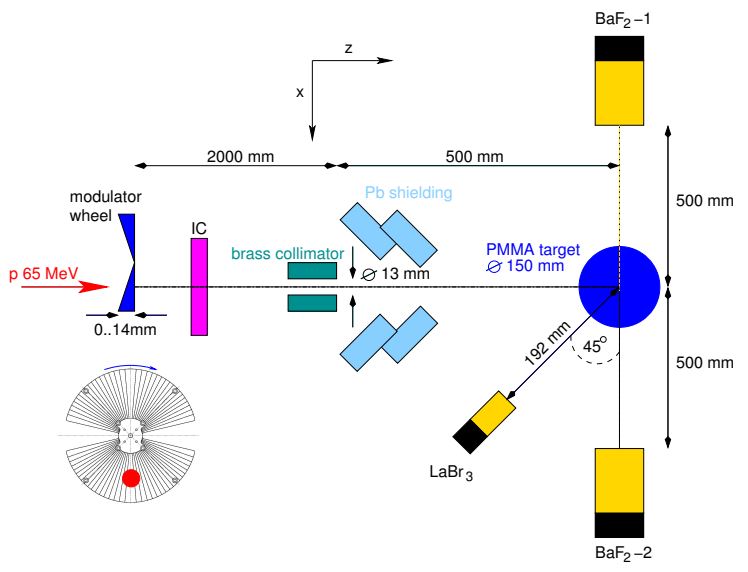

FIG. 1. Configuration for the measurements at CAL, including a sketch of the used modulator wheel. The red dot marks the beam impact at a given wheel position.

$25.4 \times 72.2 \mathrm{~mm}^{3}$ ) and $\mathrm{BaF}_{2}$ (hexagonal shape with $50 \mathrm{~mm}$ edge length and a length of $140 \mathrm{~mm}$ ). The detectors were placed under 90 or 45 degrees, with respect to the beamline, to reduce background events, e.g. neutrons which are mainly generated towards forward directions. For a suppression of the detection of events generated in elements of the beamline, the detectors were shielded via lead bricks, as indicated in the figure.

A dedicated data-acquisition (DAQ) card has been developed, which allows for the connection of up to three detectors. The front-end electronics part for each detector contains a comparator for the timing information and a shaper (1 us shaping time) for the energy information. After digitization and treatment via a FPGA, the stream with the numerical pulse height and timing data is transferred to a standard PC via an ethernet connection.

The reference signal for the TOF measurements is connected via an additional input. In the present case the high frequency signal of the accelerator is used for this purpose, but also signals from monitor detectors ${ }^{18}$ intercepting the beam are compatible. Furthermore, information about the position of the rotating modulator wheel is provided via a photo sensor. The system is designed to process rates of several $10^{5}$ counts per second without significant dead-time.

During the experiment only a single input channel of the DAQ card was operational. The experimental data which will be shown in the following section are coming from the $\mathrm{LaBr}_{3}$ detector which has been connected to this channel. The $\mathrm{BaF}_{2}$ detectors will be included in 
simulations for the discussion about the clinical applicability.

\section{RESULTS}

TOF spectra from the $\mathrm{LaBr}_{3}$ detector are shown in Figure 2. The two spectra correspond to different positions of the rotating modulator wheel. At 0 degree the beam is hitting the part of the modulator with the maximum thickness, whereas at 90 degree it is traversing no material. The proton ranges in the PMMA target correspond to $14 \mathrm{~mm}$ and $28 \mathrm{~mm}$ for these two cases, respectively. The peak in the measured spectra (black curves) is due
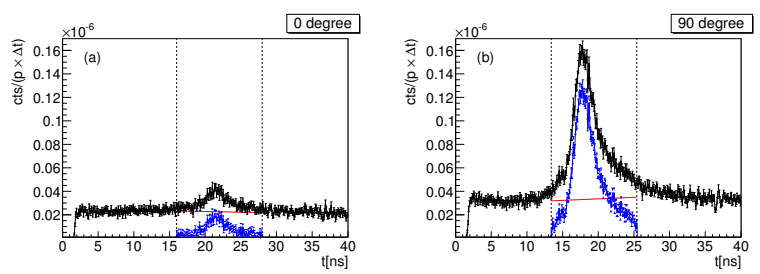

FIG. 2. TOF spectra corresponding to different positions of the modulator wheel. (a) 0 and (b) 90 degree correspond to the maximum thickness and the hole, respectively. The ordinate displays the counts per incident proton and time bin $\Delta t=0.1 \mathrm{~ns}$. The spectra contain only events above an energy threshold of $1 \mathrm{MeV}$. After subtracting the background (in red), the signals (in blue) corresponding to prompt gamma-ray emission from the target are integrated within the limits given by the vertical dashed lines.

to prompt gamma-ray production in the PMMA target. Contributions from the nozzle are largely suppressed by the lead shielding (cf. Fig. 1). The remaining background can be attributed to the generation of neutrons. The displayed spectra contain only events above an energy threshold of $1 \mathrm{MeV}$. The background is obtained from a linear interpolation (red line in Fig. 2) of the events left and right to the peak. After background subtraction, the signal (blue curves) is integrated within the limits denoted by the vertical dashed lines.

A degradation of the proton energy via the modulator leads to larger TOF values (of the protons) which is also reflected in the prompt gamma-ray spectra. In Fig. 2 the mean TOF value of the peak is shifted by 2-3 ns between 90 and 0 degree of the modulator wheel.

The information accessible from the experiment is the detected PGPI count rate and the position of the modulator wheel. These quantities are plotted in Figure 3 where the 
count rate has been normalized to the maximum value at 90 degree. For an interpretation of the data Geant4 ${ }^{19}$ simulations (version 10.02.p01 physics list QGSP_BIC_HP) have been performed which include the structures of the beamline and which also use realistic beam parameters.

The experimentally observed decrease of the detected count rate with the modulator

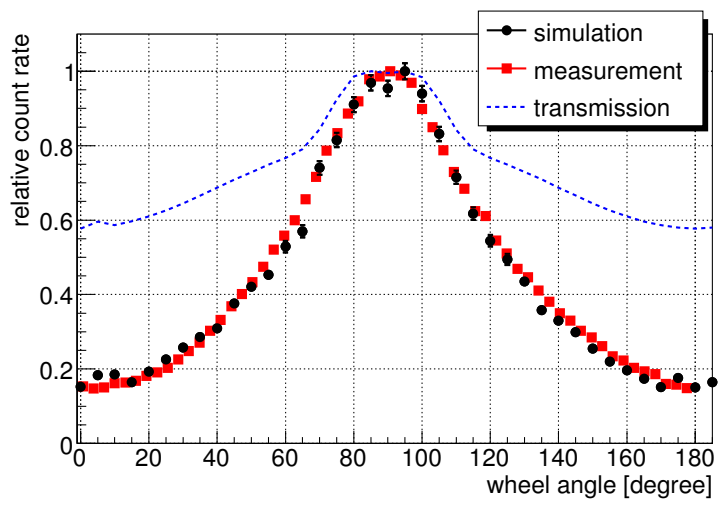

FIG. 3. Detected relative prompt gamma-ray count rates from measurement and simulation, as well as the simulated transmission through the collimator (dashed line).

thickness by more than a factor of five is well reproduced by the simulations. This decrease has two reasons: first, the reduction of the proton path inside the PMMA target, hence a reduction of the produced prompt gamma-ray rate due to the degradation of the proton energy. Second, a reduced transmission through the collimator due to scattering of the incident protons in the modulator wheel. The transmission is defined as the ratio of the number of protons traversing the collimator and hitting the PMMA target, divided by the number of protons registered by the IC. The transmission curve has been obtained from simulations and is plotted as dashed line in Fig. 3. It is also normalized to the maximum at 90 degree.

The data have been taken at a proton rate of $3 \times 10^{9} \mathrm{p} / \mathrm{s}$ on the target, which corresponds to a standard intensity of the accelerator in clinical use. The data taking rate with the $\mathrm{LaBr}_{3}$ detector was $400 \mathrm{kHz}$ at this beam intensity. 


\section{DISCUSSION: CLINICAL APPLICABILITY}

In the previous section it has been shown, that the PGPI is in principle sensitive to a variation of the primary energy via a modulator wheel, leading to a variation of the proton range inside the target. Here, it should be investigated to what extent range deviations can be detected under clinical conditions. To this end simulations have been performed where the modulator wheel has been replaced by PMMA degraders with different thicknesses. It will also be studied how different primary proton energies and different target geometries alter the results. The simulations with $65 \mathrm{MeV}$ proton energy have been performed with inclusion of the beamline elements from CAL and the detector and target configuration as indicated in Figure 1. As the beamline is not designed for higher energies, the simulations for 130 and $200 \mathrm{MeV}$ have been done with a simplified configuration consisting of a pencil beam, the degrader and a collimator. The positions of these elements, as well as the positions of the target and the detectors are identical for all proton energies. The target geometries for the higher energies consist of a PMMA cylinder with a diameter of $150 \mathrm{~mm}$, oriented along the z-axis, and a rectangular parallelepiped (RPP) with a diagonal equal to the cylinder diameter. To allow for similar conditions for the higher beam energies, the target length has been set to 1.5 times the range for protons in PMMA. The ranges for protons correspond to 106 and $223.5 \mathrm{~mm}$ for 130 and $200 \mathrm{MeV}$, respectively ${ }^{20}$.

The results are indicated in Figure 4. For an improvement of the statistics, the events registered by the two $\mathrm{BaF}_{2}$ detectors are summed up. Different colors represent the three beam energies, open and closed symbols indicate the different target geometries. For a comparison of the different beam energies the data are plotted as a function of the relative degrader length which is defined as the degrader length divided by the proton range for the respective energy. The relative count rates are normalized to the PGPIs from the RPP targets (for the higher energies) without degrader. The decrease shows a similar trend for the different proton energies. A comparison between RPP and cylindrical targets reveals a difference on the order of $8 \%$ which is due to absorption of the prompt gamma rays in the target. The absorption will be studied in more detail further down in the text.

The shown statistics corresponds to $10^{8}$ protons hitting the target. This number corresponds to a single spot at a distal energy slice for a tumor treatment with active beam delivery and pencil beam scanning $(\mathrm{PBS})^{6,21,22}$. In the case of $65 \mathrm{MeV}$ protons the relative 


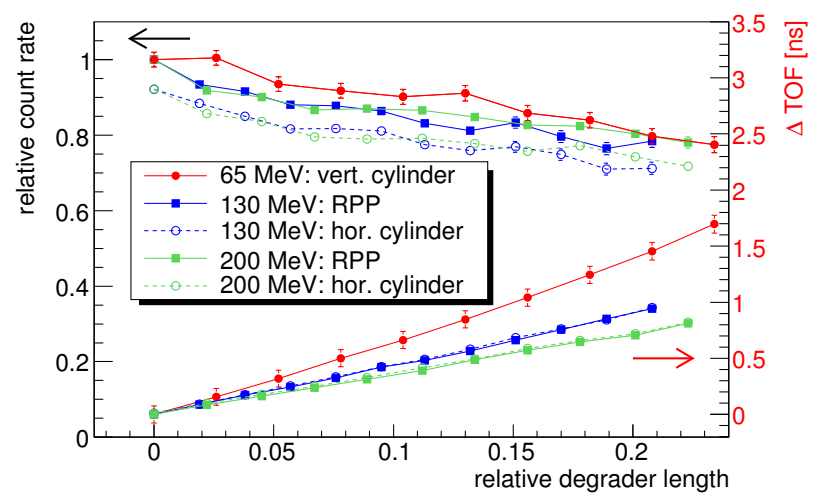

FIG. 4. Simulated relative count rates and $\triangle \mathrm{TOF}$ values of the $\mathrm{BaF}_{2}$ detectors as a function of the relative degrader length. The different beam energies are represented by different colors and the different target shapes are indicated by closed and open symbols, respectively. The arrows indicate the corresponding y-axis. The statistics corresponds to $10^{8}$ protons hitting the target.

degrader length needs to change by 0.1 in order to detect a change in the relative count rate larger than two sigma. In this case the range changes by $3 \mathrm{~mm}$, i.e. it is feasible to detect such changes with $10^{8}$ protons and detectors with reasonable sizes (diameter 3-4 inches). The other quantity indicated in Figure 4 is $\triangle \mathrm{TOF}$ which is the difference of the TOF to a reference value. As reference the values obtained with the RPP target (for the higher energies) and without degrader are used. The open and filled symbols are overlapping, indicating that the target shape has no influence on this observable. The information from the TOF is complementary to the PGPI. If a deviation from the predicted PGPI is detected, the mean TOF information helps to confine the cause for the deviation. In the present case, degraders have been used for a variation of the proton energy impinging on the target. This leads to the observed variations in the mean TOF and PGPI data. If, however, only the total number of applied protons would change, the mean TOF remains constant, whereas the PGPI would follow the number of protons.

The study with different target shapes showed that absorption of the prompt gammarays in the target plays a role. Furthermore, a displacement of the target towards or away from the detector would alter the registered prompt gamma-ray count rate, caused by the difference in the absorption. This effect is of the same order of magnitude as a possible deviation to be detected. 
For a study of the absorption, simulations with eight detectors placed homogeneously around a spherical target (20 cm diameter) have been performed with $160 \mathrm{MeV}$ protons. The target has been displaced in horizontal x-direction, whereas the detectors remained fix. Figure 5 gives the results as a function of the target position. The upper part of the figure shows that the count rates of the individual detectors vary with the target position, whereas the mean values (arithmetic or geometric) remain constant. It should be pointed out that beyond a displacement of $60 \mathrm{~mm}$ the protons are not stopped completely anymore in the target, which leads to the decrease in the count rate. For the lower part of the figure, signals of four detectors have been grouped together, and ratios have been calculated afterwards. The ratio of the left and right groups (in red) reveals a correlation with the target displacement, whereas the up/down ratio remains constant. A change of the incident proton energy to $63 \mathrm{MeV}$ (open symbols and dashed lines) has only a marginal influence on these ratios.
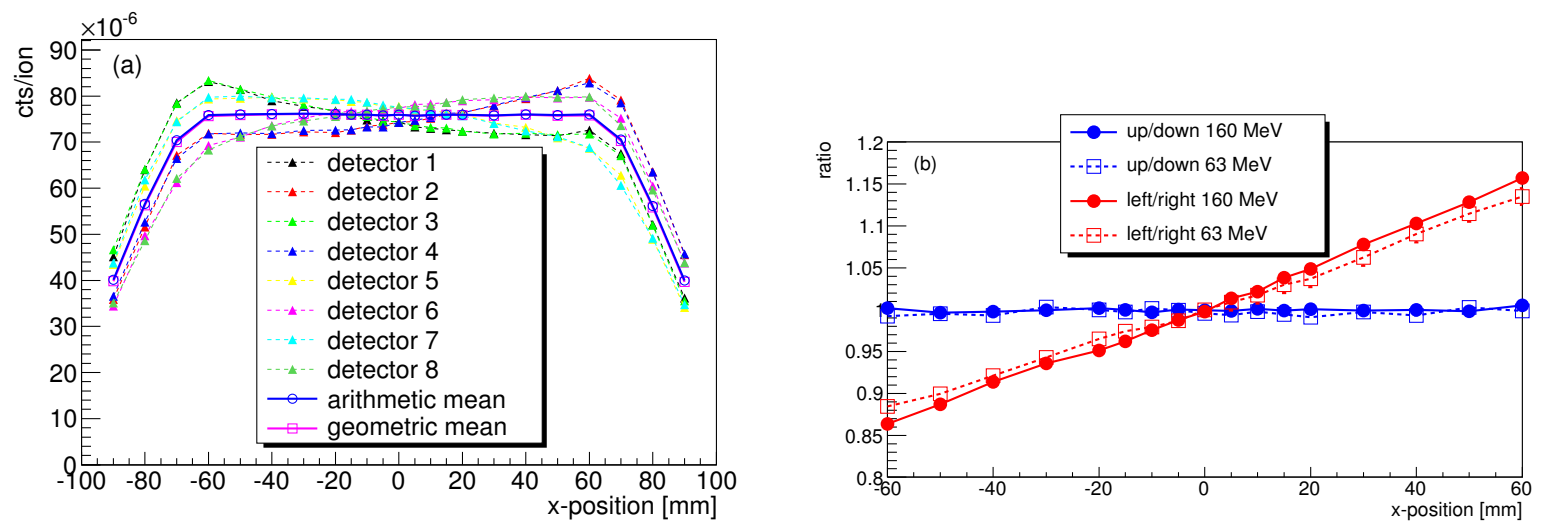

FIG. 5. Simulations with 160 (63) MeV protons. The abscissas denote the target displacement in x-direction. (a) count rates of the individual detectors, as well as the mean values for $160 \mathrm{MeV}$ protons. (b) ratios of detector groups (horizontal and vertical) for $160 \mathrm{MeV}$ (closed symbols) and $63 \mathrm{MeV}$ (open symbols) protons.

The functionality of using PGPI for monitoring purposes has been presented for homogeneous PMMA targets. For the integration of such a system in the clinical workflow, further development is required. From the mechanical point of view there should be no major caveat, as the size of the required detectors is on the order of 3-4 inches only, and no heavy collimators are needed. The detectors could be mounted directly on a gantry which would define their position in space so that no additional calibration would be necessary. For the 
prediction of the expected signal, prompt gamma-ray production needs to be integrated in treatment planning systems (TPS). So far, (accelerated) Monte Carlo techniques are used to generate prompt gamma-ray distributions for real patient data ${ }^{23}$. Improvements are required to improve the precision of these predictions to the per-cent level.

\section{CONCLUSION}

It has been shown that the PGPI method is sensitive to detect deviations of a few per cent in the registered prompt gamma-ray rate. The necessary statistics is obtained with $10^{8}$ incident protons, which corresponds to a single spot in PBS, and detectors covering a solid angle of 25 msr. The measurements, performed with a dedicated DAQ card under clinical intensities at CAL, are in accordance with Geant4 simulations.

The signals of multiple detectors can either be combined as ratios to obtain information about the target placement, or as (geometric) mean, which is independent on the actual target position.

In the present study the influence of a variation on the proton range and the absorption on the PGPI has been studied in homogeneous PMMA targets. In a forthcoming article, the influence of different target materials will be discussed.

The herein presented system is intended to be used as a simple and independent monitoring device for particle therapy.

\section{ACKNOWLEDGMENTS}

This work has been partly supported by the Gammadosi project (Inserm: Plan Physique Cancer), ANR France Hadron (ANR-11-INBS-0007), the European FP7 project ULICE (grant agreement nr. 228436) and the Pôle-Emploi. This work has been performed within the LABEX PRIMES (ANR-11-LABX-0063).

\section{REFERENCES}

${ }^{1}$ G. Shakirin, H. Braess, F. Fiedler, D. Kunath, K. Laube, K. Parodi, M. Priegnitz, and W. Enghardt, Phys. Med. Biol. 56, 1281 (2011).

${ }^{2}$ C.-H. Min, C. H. Kim, M.-Y. Youn, and J.-W. Kim, Appl. Phys. Lett. 89, 183517 (2006). 
${ }^{3}$ E. Testa, M. Bajard, M. Chevallier, D. Dauvergne, F. L. Foulher, N. Freud, J. Létang, J. Poizat, C. Ray, and M. Testa, Nucl. Instrum. Methods Phys. Res. B 267, 993 (2009). ${ }^{4}$ J. Verburg and J. Seco, Phys. Med. Biol. 59, 7089 (2014).

${ }^{5}$ M. Testa, C. Min, J. Verburg, J. Schmann, H.-M. Lu, and H. Paganetti, Physics in Medicine and Biology 59, 4181 (2014).

${ }^{6}$ J. Smeets, F. Roellinghoff, D. Prieels, F. Stichelbaut, A. Benilov, P. Busca, C. Fiorini, R. Peloso, M. Basilavecchia, T. Frizzi, J. C. Dehaes, and A. Dubus, Phys. Med. Biol. 57, 3371 (2012).

${ }^{7}$ S. Kurosawa, H. Kubo, K. Ueno, S. Kabuki, S. Iwaki, M. Takahashi, K. Taniue, N. Higashi, K. Miuchi, T. Tanimori, D. Kim, and J. Kim, Curr. Appl. Phys. 12, 364 (2012).

${ }^{8}$ J. C. Polf, S. Avery, D. S. Mackin, and S. Beddar, Phys. Med. Biol. 60, 7085 (2015).

${ }^{9}$ P. G. Ortega, I. Torres-Espallardo, F. Cerutti, A. Ferrari, J. E. Gillam, C. Lacasta, G. Llosá, J. F. Oliver, P. R. Sala, P. Solevi, and M. Rafecas, Phys. Med. Biol. 60, $1845(2015)$.

${ }^{10}$ M. Pinto, D. Dauvergne, N. Freud, J. Krimmer, J. M. Letang, C. Ray, F. Roellinghoff, and E. Testa, Phys. Med. Biol. 59, 7653 (2014).

${ }^{11}$ C. Richter, G. Pausch, S. Barczyk, M. Priegnitz, I. Keitz, J. Thiele, J. Smeets, F. V. Stappen, L. Bombelli, C. Fiorini, L. Hotoiu, I. Perali, D. Prieels, W. Enghardt, and M. Baumann, Radiotherapy and Oncology 118, 232 (2016).

${ }^{12}$ C. Golnik, F. Hueso-González, A. Müller, P. Dendooven, W. Enghardt, F. Fiedler, T. Kormoll, K. Roemer, J. Petzoldt, A. Wagner, and G. Pausch, Phys. Med. Biol. 59, 5399 (2014).

${ }^{13}$ F. Hueso-González, W. Enghardt, F. Fiedler, C. Golnik, G. Janssens, J. Petzoldt, D. Prieels, M. Priegnitz, K. E. Roemer, J. Smeets, F. V. Stappen, A. Wagner, and G. Pausch, Phys. Med. Biol. 60, 6247 (2015).

${ }^{14}$ J. Petzoldt, K. E. Roemer, W. Enghardt, F. Fiedler, C. Golnik, F. Hueso-González, S. Helmbrecht, T. Kormoll, H. Rohling, J. Smeets, T. Werner, and G. Pausch, Phys. Med. Biol. 61, 2432 (2016).

${ }^{15}$ S. Goetsch, http://sdradiotherapy.com/Packages.html (2013).

${ }^{16}$ A. Carnicer, V. Letellier, G. Rucka, G. Angellier, W. Sauerwein, and J. Hérault, Med. Phys. 39, 7303 (2012).

${ }^{17}$ J.Hérault, N. Iborra, B. Serrano, and P. Chauvel, Med. Phys. 32, 910 (2005). 
${ }^{18}$ M.-L. Gallin-Martel, A. Bes, A. Boukhémiri, G. Bosson, J. Collot, D. Dauvergne, M. Fontana, L. Gallin-Martel, A. Gorécki, J.-Y. Hostachy, J. Krimmer, A. Lacoste, J. Morse, J.-F. Muraz, F. Rarbi, M. Salome, E. Testa, M. Yamouni, and Y. Zoccarato, 2016 IEEE NSS/MIC conference, Strasbourg, France (2016).

${ }^{19}$ S. Agostinelli, , J. Allison, K. Amako, J. Apostolakis, H. Araujo, P. Arce, M. Asai, D. Axen, S. Banerjee, G. Barrand, et al., Nucl. Instrum. Methods Phys. Res. B 506, 250 (2003). ${ }^{20} \mathrm{PSTAR}$, http://physics.nist.gov.

${ }^{21}$ M. Krämer, O. Jäkel, T. Haberer, G. Kraft, D. Schardt, and U. Weber, Phys. Med. Biol. 45, 3299 (2000).

${ }^{22}$ L. Grevillot, D. Bertrand, F. Dessy, N. Freud, and D. Sarrut, Phys. Med. Biol. 56, 5203 (2011).

${ }^{23}$ B. F. B. Huisman, J. M. Létang, E. Testa, and D. Sarrut, Phys. Med. Biol. 61, 7725 (2016). 